\title{
Structure and Function of Hornwort Stomata
}

\author{
Jessica R. Lucas and Karen S. Renzaglia
}

Department of Plant Biology, Southern Illinois University, Carbondale, IL 62901

As minute structures that open and close in response to environmental cues, stomata are key adaptive innovations of land plants [1]. In vascular plants, stomata function in gas exchange in photosynthetic tissue and in facilitating nutrient transport through the transpirational stream. In bryophytes, the basal most land plants, stomata are restricted in occurrence and are poorly characterized in regards to structure-function relationships. This study was undertaken to evaluate the microanatomy and physiology of stomata in hornworts, the oldest living lineage of plants that possess stomata. Protocols for SEM and TEM follow Renzaglia et al. [2]. To assess the osmotic regulation of stomatal functioning, we performed cytochemical localizations of potassium with Macullum's reagent [3] and organic ions with Fast Violet B solution [4].

In hornworts, stomata occur scattered among elongated epidermal cells in the sporophyte. Mature stomata each consist of two bean-shaped guard cells subtended by an air-filled cavity (Figs. 1-3). In cross section, guard cells are larger than epidermal cells and they each contain a large starchfilled chloroplast (Figs. 1, 2). Aside from prominent ledges on the outer and inner walls where the cells meet, the guard cell wall is thin throughout (Fig. 1). Extensive fibrous and flocculent material is associated with the outer ledge where the pore forms (Figs. 1, 3). The large chloroplasts in the underlying photosynthetic tissue are invariably positioned adjacent to air spaces, indicating a role of stomata in gas exchange (Fig. 4). In contrast, the lack of an internal conducting system in hornworts suggests that stomata are not involved in nutrient transport or in regulating the transpirational flow as they are in plants with vascular tissue.

Stomata examined in immature regions of the sporophyte are closed, while in mature regions, the stomata are typically opened. Examination of sporophytes collected during dark and light periods indicates that there are no diurnal movements, i.e., guard cells in hornworts do not open and close in response to light. Further experiments with abscisic acid, a hormone that results in stomatal closure in vascular plants, resulted in no change in the pore (not illustrated). In immature regions, both $\mathrm{K}+$ and malate exhibit general localization in all epidermal cells (Figs. 5, 7). In mature tissue, these ions are localized in guard cells and are interpreted as functioning as osmoticum that is responsible for water influx and the creation and maintenance of stomatal pores (Figs. 6, 8). These results coupled with the structural observations above support the concept that hornwort stomata open once through osmotic changes and remain open during the duration of physiological activity of the sporophyte. It appears that unlike vascular plants, the primary role of stomata in hornworts is in providing a passageway for gas exchange.

[1] E. Zeigler et al. 1987. Stomatal Function. Stanford Univ. Press, Stanford, CA.

[2] K.S. Renzaglia et al. 2001. Amer. J. Bot. 88:1151-1163.

[3] K. Raschke \& M.P. Fellows. 1971. Planta 101: 296-316.

[4] B.A. Palevitz et al. 1981. Protoplasma 109: 23-55.

[5] This research was supported by NSF grant DEB-9527735 (REU Program) and undergraduate research scholarships from the Microscopy Society of America and the Illinois State Academy of Science. 


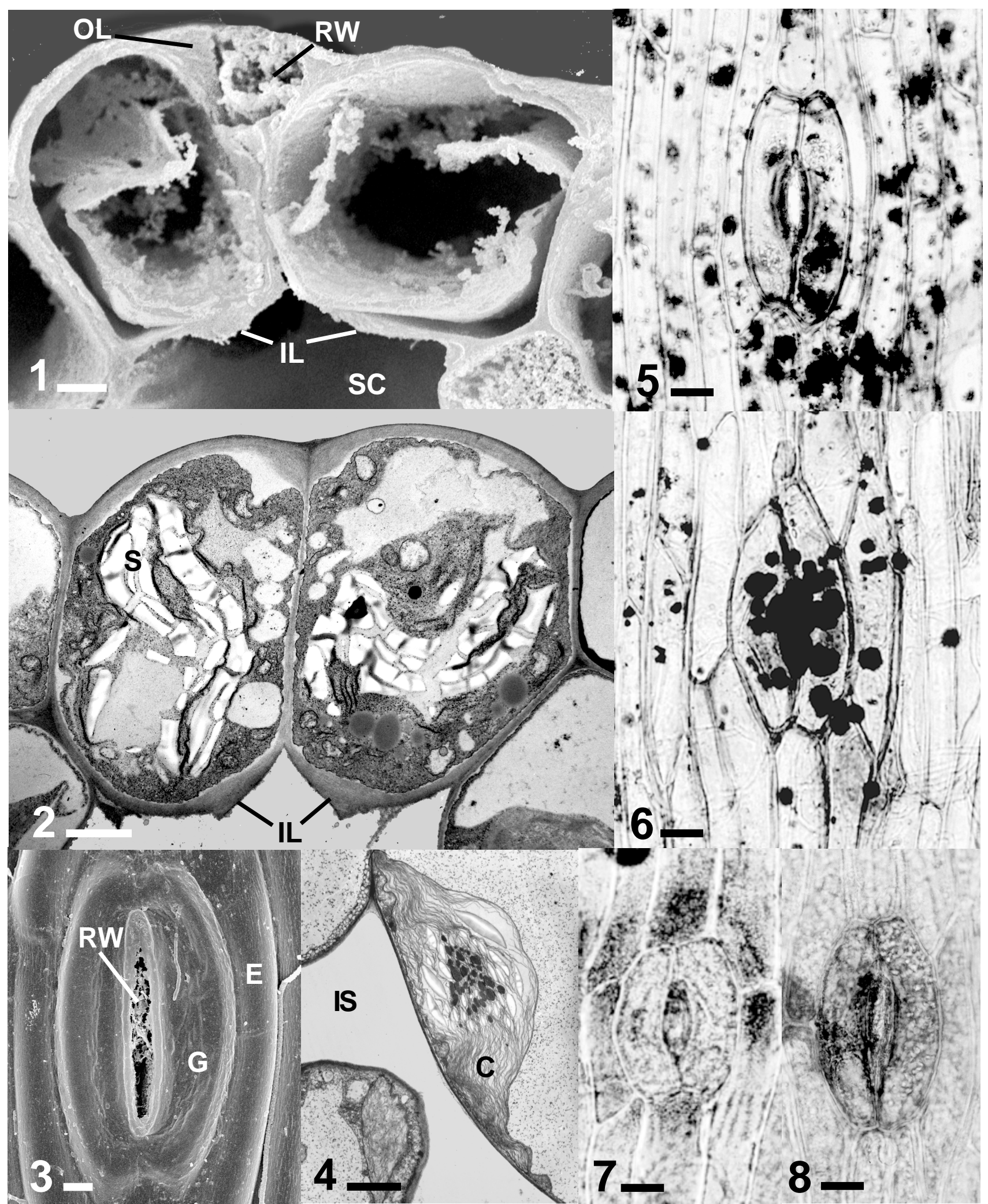

Figure 1. Phaeoceros carolinianus. SEM cross section of stoma. Bar $=5 \mu \mathrm{m}$. Figure 2. Folioceros fuciformis. TEM transverse section. Bar $=5 \mu \mathrm{m}$. Figure 3. P. carolinianus. Surface view. Bar $=10 \mu \mathrm{m}$. Figure 4. F. fuciformis. Cross section through substomatal chamber. Bar $=5 \mu \mathrm{m}$. Figure 5,6. Anthoceros caucaicus. Staining with Macullum's reagent. Figures 7,8. P. carolinianus. Fast Violet B stain. Figures 5-8. Bars $=30 \mu \mathrm{m}$. Key to labeling; C, chloroplast; E, epidermal cell; G, guard cell; IL, inner ledge; IS, intercellular space; OL, outer ledge; RW, remnant wall; S, starch; SC, substomatal chamber. 